University of South Carolina

Scholar Commons

3-1990

\title{
The Extended Tracking Network and Indications of Baseline Precision and Accuracy in the North Andes
}

Jeffrey T. Freymueller

James N. Kellogg

University of South Carolina - Columbia, kellogg@geol.sc.edu

Follow this and additional works at: https://scholarcommons.sc.edu/geol_facpub

Part of the Earth Sciences Commons

\section{Publication Info}

Published in Geophysical Research Letters, Volume 17, Issue 3, 1990, pages 207-210.

Freymueller, J. T. \& Kellogg, J. N. (1990). The extended tracking network and indications of baseline precision and accuracy in the North Andes. Geophysical Research Letters, 17 (3), 207-210. (c) Geophysical Research Letters 1990, American Geophysical Union

This Article is brought to you by the Earth, Ocean and Environment, School of the at Scholar Commons. It has been accepted for inclusion in Faculty Publications by an authorized administrator of Scholar Commons. For more information, please contact digres@mailbox.sc.edu. 


\title{
THE EXTENDED TRACKING NETWORK AND INDICATIONS OF BASELINE PRECISION AND ACCURACY IN THE NORTH ANDES
}

\author{
Jeffrey T. Freymueller and James N. Kellogg \\ Department of Geological Sciences, University of South Carolina, Columbia
}

\begin{abstract}
The CASA UNO Global Positioning System (GPS) experiment (January-February 1988) included an extended tracking network which covered three continents in addition to the network of scientific interest in Central and South America. The repeatability of long baselines $(400-1000 \mathrm{~km})$ in South America is improved by up to a factor of two in the horizontal vector baseline components by using tracking stations in the Pacific and Europe to supplement stations in North America. In every case but one, the differences between the mean solutions obtained using different tracking networks was equal to or smaller than day-to-day Ims repeatabilities for the same baselines. The mean solutions obtained by using tracking stations in North America and the Pacific agreed at the 2-3 millimeter level with those using tracking stations in North America and Europe. The agreement of the extended tracking network solutions suggests that a broad distribution of tracking stations provides better geometric constraints on the satellite orbits and that solutions are not sensitive to changes in tracking network configuration when an extended network is used. A comparison of the results from the North Andes and a baseline in North America suggests that the use of a geometrically strong extended tracking network is most important when the network of interest is far from North America.
\end{abstract}

\section{Introduction}

The CASA UNO Global Positioning System (GPS) experiment took place in January and February of 1988. Its primary goal was to establish first epoch measurements for the long-term study of crustal deformation in the northern Andes of Colombia and adjacent areas in Venezuela, Ecuador, Panama and Costa Rica. The specific tectonic problems which will be addressed by the CASA experiments are discussed by Kellogg and Dixon [this issue] and Kellogg et al. [this issue]. Repeat measurements of this network will allow the measurement of the present rates of motion across a complex plate boundary region, including rapid subduction across the Colombia trench. In addition, the CASA UNO data set provided the first opportunity to analyze the sensitivity of GPS geodetic results to the configuration of an extended tracking network spanning several continents.

This study focuses on the effect of the extended tracking nerwork on repeatability and mean solutions for several baselines of geophysical interest in the North Andes, ranging in length from $100 \mathrm{~km}$ to $1300 \mathrm{~km}$. We compared four subsets of the CASA UNO extended tracking network: (a) North American tracking stations only; (b) North American and European tracking stations; (c) North American and Pacific tracking stations; and (d) the entire extended tracking network.

The GPS satellite constellation at the time of the CASA UNO experiment consisted of 7 satellites, optimized for tracking in western North America. As viewed from the North Andes, the constellation was far from optimal as the satellites often remained at low elevation angles and four or more satellites were visible simultaneously for only a short time. During

Copyright 1990 by the American Geophysical Inion.

Paper number 90GL00417.

0094-8276/90/90GL-00417\$03.00
CASA UNO, the daily observation periods were long (7.5 or 9 hours) to compensate for the poor geometry.

The GPS satellite orbits must be estimated along with station locations when the desired baselines are longer than a few tens of kilometers in length. The fiducial network approach was developed to improve the estimation of satellite orbits and to provide a consistent reference frame. We implement the fiducial network as described in Davidson et al. [1985]. Three stations whose positions are known from very long baseline interferometry (VLBD) are held fixed while the remaining station locations are estimated. For the CASA UNO experiment, several tracking stations outside of North America were used in addition to the three fixed fiducial stations.

A covariance study by Freymueller and Golombek [1988] predicted that the use of an extended fiducial network would result in a small improvement in precision for baselines up to $500 \mathrm{~km}$ (longer baselines were not considered), and that a geometrically strong network could reduce the sensitivity of GPS baselines to certain systematic errors. In general, systematic errors will be different from experiment to experiment unless the same network, satellite constellation, and observation schedules are used. Larger improvements would be expected for baselines located farther away from North America [Wu et al., 1988]. In both of these previous studies all of the tracking stations were held fixed. However, fixing all the tracking stations could potentially increase the systematic errors affecting the baseline results if the locations of all the stations held fixed were not consistent at the centimeter level [Freymueller and Golombek, 1988]. In the analysis described here the locations of the extended tracking stations were estimated along with the locations of the stations of geophysical interest.

\section{Method of Analysis}

The GIPSY software [Stephens, 1986; Lichten and Border, 1987; Sovers and Border, 1988], developed at Jet Propulsion Laboratory (JPL), was used to analyze the CASA UNO data. The GIPSY software uses undifferenced data and estimates receiver and satellite clocks as white noise, an approach which is equivalent to double-differencing. Other parameters may be estimated as constants, as white noise, or as Gauss-Markov process noise [Lichten and Border, 1987]. Vector baselines are derived from estimates of the station locations.

For this study, four subsets of the extended tracking network (Figure 2, Kellogg and Dixon, this issue) were considered. Network (a) used only North American tracking stations: Mojave and Owens Valley (CA), Westford (MA), and Ft. Davis (TX). Network (b) consisted of two stations in Europe, Onsala (Sweden) and Wettzell (W. Germany), added to the North American network For network (c), two stations in the Pacific, Canberra (Australia) and Black Birch (New Zealand), were added instead of stations in Europe. Network (d) consisted of the full tracking network, using all stations listed above. Data from the same ten stations in the North Andes (Table 1, Kellogg et al., this issue; Figure 1, Kellogg and Dixon, this issue) was used with all tracking networks studied. Data from the first two weeks of the CASA UNO experiment were used. Each solution used one day of data, making the solutions completely independent.

The same three stations, Owens Valley, Ft. Davis, and Westford, were held fixed in every solution except for one day 
(Jan. 18) on which there were no observations for Owens Valley and it was replaced with Mojave. When they were used in the solution, the additional stations in the extended tracking network were estimated without constraints. Tropospheric delays were estimated as Gauss-Markov process noise [Lichten and Border, 1987; Tralli et al., 1988]. Canier phase biases were resolved using the method of Blewitt [1989] using a confidence level of 0.995 , which reflects the cumulative probability that the entire set of fixed biases were fixed to the correct values. In the network as a whole, including North America, $62 \%$ of the double-differenced biases were resolved over baselines ranging up to $1600 \mathrm{~km}$ in length. Within the subset of the network on continental South America, $75 \%$ of the double-differenced biases were resolved. During the second week, with better data quality, $90 \%$ of the double-differenced biases on continental South America were resolved.

\section{Results}

Daily rms repeatabilities of the estimated horizontal baseline components are shown in Figure 1 for the North America only network and the full network. Sixteen baselines which were observed at least four times are shown. Using the full extended tracking network resulted in better repeatability than using only North American tracking stations, although the improvement is small for most baselines shorter than about 300 $\mathrm{km}$. The superiority of the full tracking network solution is evident in the repeatabilities of baselines between 400 and 1000 $\mathrm{km}$. For baselines in this range of lengths, the observed repeatabilities are independent of baseline length for all tracking networks. Mean values are given in Table 1. For the two very long baselines $(>1000 \mathrm{~km})$, no improvement in repeatability is seen with the full network. For both of these very long baselines the rms repeatability of the baseline components are considerably lower than the formal errors for all tracking networks. A larger set of very long baselines is needed before any conclusions can be drawn. Baseline vertical rms repeatabilities (not shown) range between 20 and 50 millimeters, with short baselines usually having better vertical repeatability. Vertical repeatabilities do not appear to be significantly affected by the tracking network used.

TABLE 1. Mean rms repeatabilities for baselines between 400 and $1000 \mathrm{~km}$ in length (millimeters).

\begin{tabular}{lcc}
\hline Tracking Network & East & North \\
\hline NA Only & 33 & 19 \\
NA + Europe & 32 & 19 \\
NA + Pacific & 27 & 18 \\
Full tracking net. & 21 & 12 \\
\hline
\end{tabular}

Certain baselines have repeatabilities which are much larger than would be predicted by a linear increase in ms repeatability with baseline length; in every case the large rms repeatabilities are attributable to problems with one of the stations and are in accordance with the formal errors for those baselines. The worst stations were at Malpelo Island, where a mountain blocked visibility in the westem sky so that four satellites were not viewed simultaneously on most days, and Isla La Palma, where heavy jungle growth obscured the satellite signals at low elevations. The baselines with lengths between 400 and $1000 \mathrm{~km}$ connect Malpelo with stations on the mainland. Isla La Palma is represented by two baselines with lengths of approximately 100,300 and $500 \mathrm{~km}$.

Figure 2 shows a comparison of the mean solutions using the four subsets of the extended tracking network for the baseline between Bogota and Cali $(294 \mathrm{~km})$, based on five days of data. This baseline crosses the Romeral fault zone, a (a) Only North America tracking

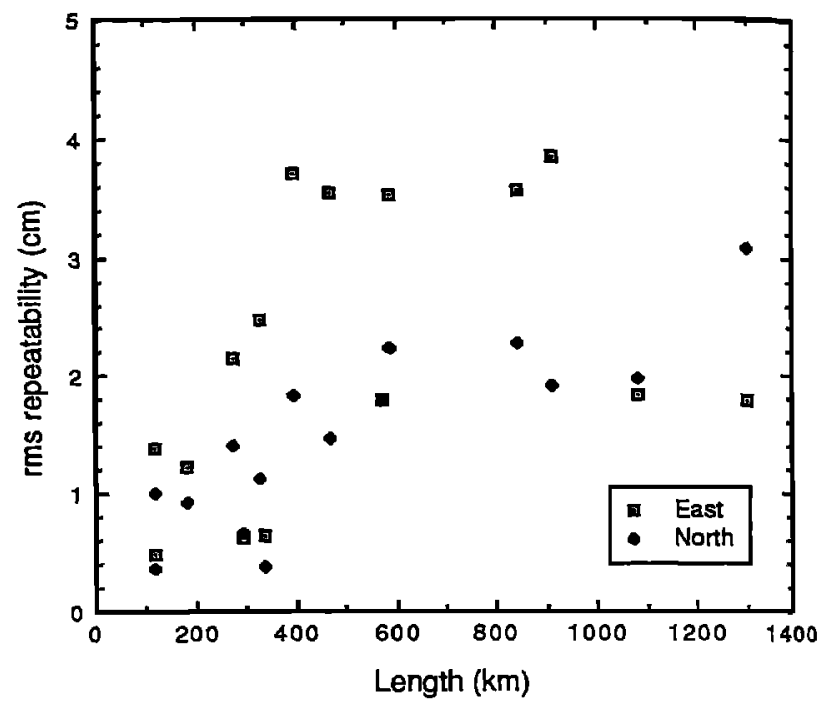

(b) Full tracking network

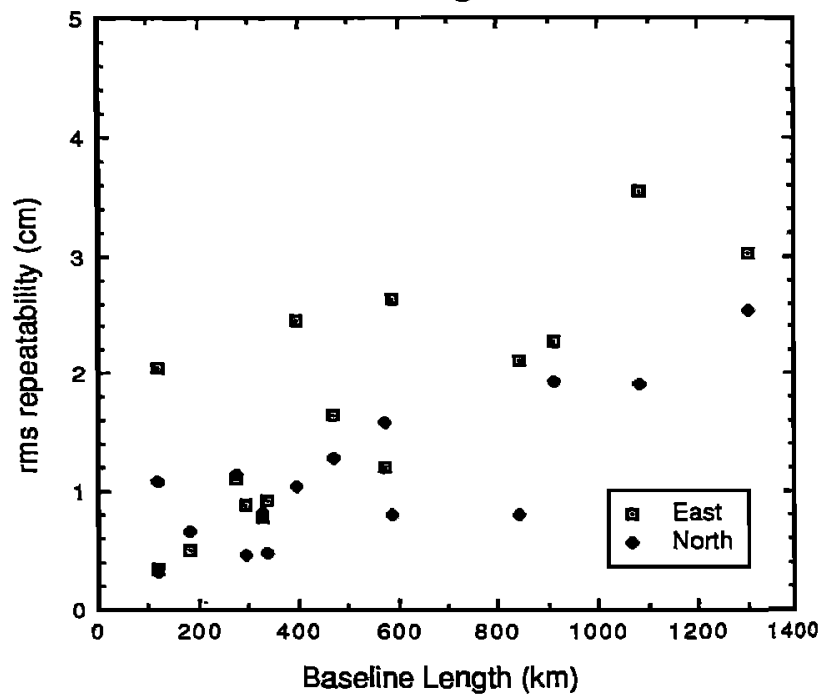

Fig. 1. Daily rms repeatability of sixteen baselines as a function of baseline length for each subset of the tracking network: (a) North America only, (b) Full tracking network. In neither of the cases does the observed repeatability scale linearly with baseline length; station-dependent effects appear to be more significant (see text).

seismically active fault system in western Colombia. The differences between the mean solutions are the same or smaller than the rms repeatabilities for each network. Indicated by a star is the mean solution for an additional case in which two additional stations in the Pacific, Kokee (Hawaii) and Samoa are added to the full network. The addition of these two stations changes the solution by more than 5 millimeters, and this solution does not agree with the solutions from all the other cases. The results from this and other baselines in South and Central America [Kornreich Wolf, personal communication] suggest that the addition of Kokee and Samoa introduced a bias into the baseline solutions.

Table 2 shows the mean solutions for the well-studied baseline between Mojave and OVRO in Califomia, taken from the same solutions as were shown above. Estimates are expressed as offsets in millimeters from the full network solvtion, and repeatabilities are also given in millimeters. There is 


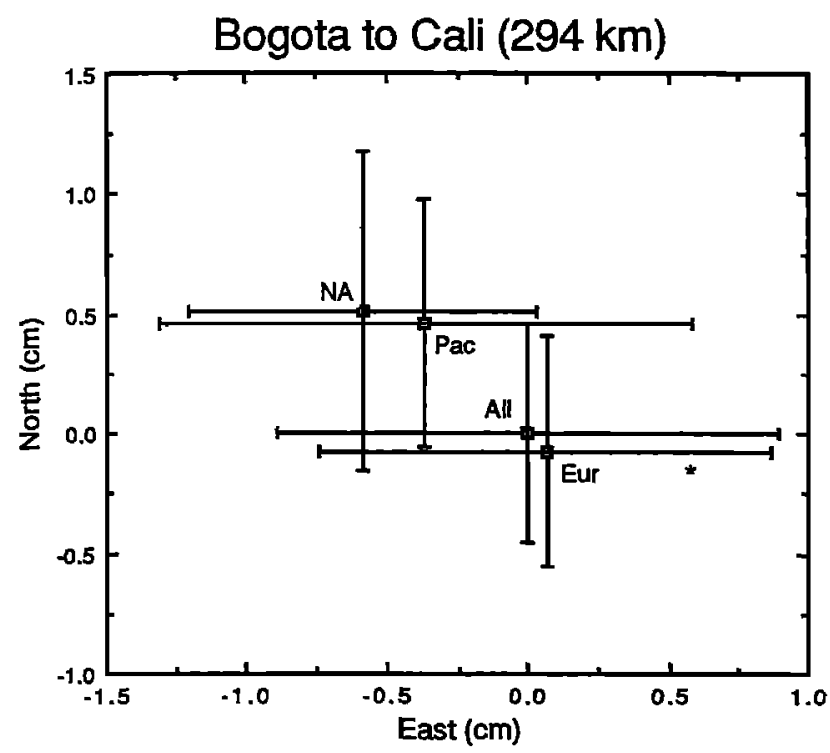

Fig. 2. Mean baseline solutions based on five days of data, using the four subsets of the extended tracking network: North America-only (NA), North America and Europe (Eur), North America and Pacific (Pac), and the full network (All). The daily rms repeatability of solutions is indicated by the error bars. The differences between these mean solutions are equal to or smaller than the repeatabilities. The star $\left({ }^{*}\right)$ indicates the mean solution when the stations Kokee (Hawaii) and Samoa are added to the full network.

TABLE 2. Mean solutions for Mojave to Owens Valley baseline using different tracking networks.

\begin{tabular}{lrrr}
\hline Tracking Network & East & North & \multicolumn{1}{c}{ Vertical } \\
\hline NA Only & $3 \pm 4$ & $-3 \pm 2$ & $-18 \pm 29$ \\
NA + Europe & $2 \pm 3$ & $0 \pm 2$ & $-2 \pm 25$ \\
NA + Pacific & $2 \pm 3$ & $-1 \pm 2$ & $-5 \pm 24$ \\
Full tracking net. & $0 \pm 2$ & $0 \pm 2$ & $0 \pm 30$ \\
Full + Kokee and Samoa & $-1 \pm 3$ & $-3 \pm 2$ & $-52 \pm 30$
\end{tabular}

excellent agreement between the formal errors and repeatability for this baseline. All solutions agree in the horizontal components, but the addition of the two extra stations shifted the vertical solution by 50 millimeters. This change is almost twice as targe as the repeatability for that component and is far larger than the changes seen when other stations were added to the tracking network. Schutz et al. [this issue] successfully used the data from these two stations in orbit solutions with an algorithm that estimated independent zenith troposphere delays every 3 hours. Research is continuing to determine if troposphere mismodeling at one of the two stations is responsible for the bias we observed.

The differences between mean solutions for the four subsets of the extended tracking network are summarized in Figure 3. The same set of baselines are used as in the previous section. For baselines shorter than $400 \mathrm{~km}$, all networks agree to better than 6 millimeters in each component. The solutions for all three extended tracking networks are in even closer agreement In particular, the $\mathrm{mm}$ difference between the mean solutions obtained including tracking stations in the Pacific and those including stations in Europe was 2 millimeters in the baseline east component and 4 millimeters in the north for baselines up to $1000 \mathrm{~km}$ in length. There is some dependence
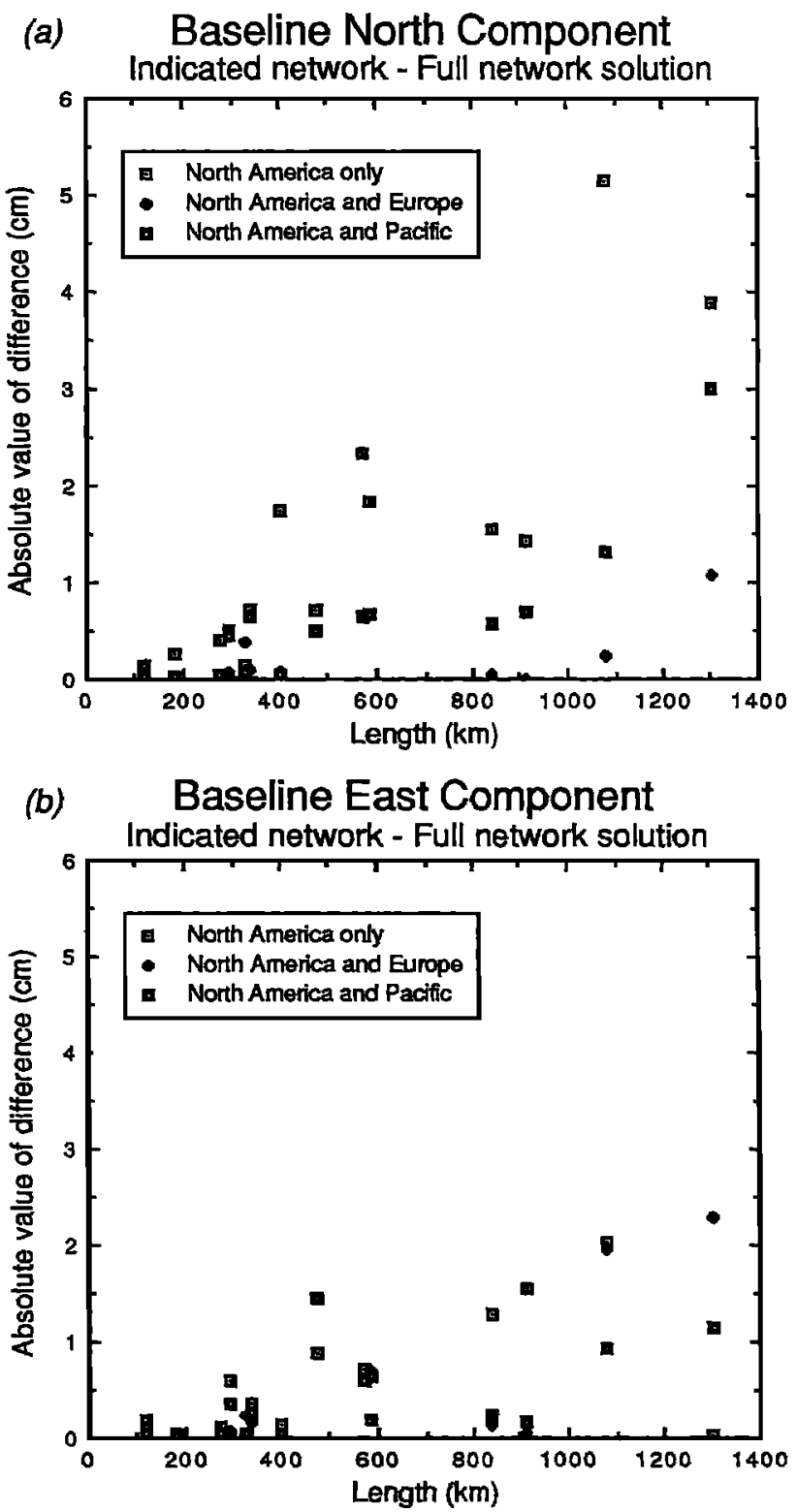

Fig. 3. Absolute values of the differences between mean solutions for baselines using a subset of the tracking network and the solution using the full tracking network: $(a)$ baseline north component, (b) baseline east component. For each component all the tracking networks agree with each other for short baselines. With one exception, all differences are equal to or smaller than the repeatability and formal errors.

of the difference on baseline length, as the longest baselines show the largest differences, but baseline-specific effects appear to be equally important. With one exception, the differences between mean solutions are smaller than the repeatabilities of those solutions. For the baseline between Baltra and Malpelo the solution using only the North American tracking stations does not agree with the others in the baseline north component.

\section{Discussion}

Baseline repeatabilities are improved by supplementing a North American tracking network with stations in Europe and the Pacific, particularly for baselines with lengths of several 
hundred $\mathrm{km}$. For a test baseline $(245 \mathrm{~km})$ within North America, all tracking networks gave essentially identical results. The extended tracking network is most important when the stations of interest are located far from the (fixed) fiducial stations and when baselines are fairly long. The results of the two very long baselines $(>1000 \mathrm{~km})$ are difficult to interpret from this study. One of the two baselines included the station at Malpelo Island, which had poor visibility. More testing with a larger set of very long baselines is needed.

The mean solutions from all three of the extended tracking networks studied in this analysis agreed to within several millimeters for baselines up to $1000 \mathrm{~km}$ in length. The mean solutions obtained including tracking stations in the Pacific and those including stations in Europe agree to better than a few millimeters on average for baselines up to $1000 \mathrm{~km}$ in length. Solutions obtained using only North American tracking stations agreed with those using the extended networks to within 25 millimeters over the same range of baseline lengths. The better agreement of the extended tracking network solutions suggests that a broad distribution of tracking stations provides better geometric constraints on the satellite orbits and that baseline solutions are not sensitive to changes in tracking network configuration when an extended network is used. Future research must determine whether this agreement is indicative of improved solution accuracy.

Acknowledgements. The suggestions and criticism of two anonymous reviewers greatly improved this paper. Support for the U. S. Participants in CASA UNO was from NASA, NSF Grant EAR-8617485, University of South Carolina, and the University NAVSTAR Consortium. Additional support for the first author was provided by an Amoco Geophysics Fellowship at the University of South Carolina and the Jet Propulsion Laboratory of the California Institute of Technology.

\section{References}

Blewitt, G., Carrier Phase Ambiguity Resolution for the Global Positioning System Applied to Geodetic Baselines up to $2000 \mathrm{~km}, J$. Geophys. Res., 94, $10,187-10,203,1989$.

Davidson, J. M., C. L. Thornton, C. J. Vegos, L. E. Young and T. P. Yunck, The March 1985 demonstration of the fiducial network concept for GPS geodesy: A prelimi- nary report, Proceedings of the First International Symposium on Precise Positioning with GPS, 603-612, 1985.

Freymueller, J., and M. Golombek, Geometry and Treatment of Fiducial Networks: Effect on GPS Baseline Precision in South America, Geophys. Res. Letters, 15, 1467. 1469,1988

Kellogg, J. N., and T. H. Dixon, Central and South America GPS Geodesy - CASA UNO, Geophys. Res. Letters, this issue, 1990.

Kellogg, J., J. Freymueller, T. Dixon, R. Neilan, C. Ropain U., S. Camargo M., B. Fernandez Ch., J. Stowell, A. Salazar, J. Mora V., L. Espin, V. Perdue, and L. Leos, First GPS Baseline Results from the North Andes, Geophys. Res. Letters, this issue, 1990.

Lichten, S. M., and J. S. Border, Strategies for High Precision GPS Orbit Determination, J. Geophys. Res., 92, 12,751-12,762, 1987.

Schutz, B. E., C. S. Ho, P. A. M. Abusali, and B. D. Tapley, CASA UNO orbit and baseline experiments, Geophys. Res. Letters, this issue, 1990.

Sovers, O. J., and J. S. Border, Observation Model and Parameter Partials for the JPL Geodetic GPS Modeling Software "GPSOMC", JPL Publication 87-21, Rev. 1. Jet Propulsion Laboratory, Pasadena, Calif., 1988.

Stephens, S. A., GIPSY Frontend User's Guide, JPL Publication JPL D-3918, Jet Propulsion Laboratory, Pasadena, Calif., 1986.

Tralli, D., T. Dixon, and S. Stephens, The Effect of Wet Tropospheric Path Delays on Estimation of Geodetic Baselines in the Gulf of California using the Global Positioning System, J. Geophys. Res., 93, 6545-6558, 1988.

Wu, S. C., W. G. Melbourne, and T. P. Yunck, Impact of Tracking Network Variation on GPS Orbit Determination, Presented at AIAA 26th Aerospace Sciences Meeting, Reno, AIAA Paper \#88-0573, 1988.

J. Freymueller and J. Kellogg, Dept. of Geological Sciences, Univ. of South Carolina, Columbia, SC 29208.

(Received: November 9, 1989;

Revised: January 31, 1990;

Accepted: February 1, 1990) 